\title{
Protée
}

\section{L'art ex situ. À propos du catalogue Kounellis au Château de Plieux.}

\section{Jan Baetens}

Volume 26, numéro 3, 1998

Logique de l'icône

URI : https://id.erudit.org/iderudit/030531ar

DOI : https://doi.org/10.7202/030531ar

Aller au sommaire du numéro

Éditeur(s)

Département des arts et lettres - Université du Québec à Chicoutimi

ISSN

0300-3523 (imprimé)

1708-2307 (numérique)

Découvrir la revue

Citer cet article

Baetens, J. (1998). L’art ex situ. À propos du catalogue Kounellis au Château de Plieux. Protée, 26(3), 100-107. https://doi.org/10.7202/030531ar
Résumé de l'article

Le catalogue d'exposition est un genre fort particulier, qui oscille en permanence entre document et monument : s'il s'agit en effet de rendre compte d'une pratique souvent éphémère, il n'est pas rare de constater que le double photographique de l'oeuvre tend à s'en faire le substitut, voire à l'effacer à tout jamais. À partir d'un exemple précis - emprunté au corpus de l'arte povera (l'exposition Kounellis à Plieux) -, cet article propose une microlecture de la façon dont l'auteur, le photographe ainsi que le metteur en pages d'un catalogue retrouvent et accentuent le geste créateur initial. 


\title{
L'ART EX SITU À PROpos du CATAlogue KOUNELLIS AU CHÂteAU DE PLIEUX
}

\author{
JAN BAETENS
}

\section{Le catalogue:}

un objet entre «document" et "monument»

L'outil photographique a été, dans ses rapports avec le monde de l'art, d'une ambiguïté sans fin. Loin de priver la création artistique de son aura particulière, comme on l'a cru un peu trop vite avec Walter Benjamin, la caméra semble au contraire avoir exacerbé - et sans doute en partie créé notre sensibilité à l'absolue et insaisissable singularité de l'œuvre, y compris l'œuvre photographique. Le culte de l'original n'est pas mort avec la diffusion des doubles photographiques et, même dans le champ des reproductions mécaniques elles-mêmes, l'effet de signature ou le prestige de l'auteur n'ont en rien été entamés.

En même temps, la photographie s'est aussi trouvée à l'origine d'une critique de l'œuvre bien plus radicale encore que la prétendue perte de l'aura. De cette mise en question, l'instance prégnante entre toutes demeure évidemment le ready made, dont le lien avec l'invention de la photographie a été remarqué par plus d'un auteur ${ }^{1}$. En effet, si l'œuvre se prête au redoublement photographique, la conséquence qu'il faut bien en tirer, c'est que tout produit ou, plus exactement encore, n'importe quel objet, trouvé ou créé, est susceptible désormais d'être lancé sur le marché de l'art (car il faut un marché, un contexte, une institution pour que la manœuvre puisse avoir un sens quelconque).

Une ambivalence comparable s'observe dans un des phénomènes qui prolongent le mieux cette intégration de l'œuvre et du contexte au sens très matériel du terme: l'art in situ. Avec cette démarche, où l'œuvre se voit déduite des propriétés d'un lieu qu'elle transforme en retour ${ }^{2}$, la même coïncidence paradoxale de destitution et de valorisation mélangées se manifeste clairement. D'un côté, le contexte jusque-là externe est imprégné par l'action créatrice de l'œuvre. De l'autre, l'effacement de l'ancienne frontière entre lieu d'accueil et production artistique, voire la substitution de l'endroit travaillé au travail de l'œuvre, inscrit non moins une dépréciation certaine de l'ancien objet d'art.

Produit dérivé devenu banal s'il en est, le catalogue d'exposition se place incontestablement au carrefour de ces interrogations. L'importance de ce genre métatextuel tient certes à de multiples raisons, qu'il serait présomptueux de vouloir creuser en ces pages ${ }^{3}$, mais l'évolution générale du monde de l'art vers la création in situ y joue un rôle sans doute fondamental quoique ambigu et paradoxal, pour ne pas dire aporétique. Car s'il n'est pas aisé de préciser ce que vaut exactement la trace d'un événement (en l'occurrence le catalogue d'une exposition) lorsque compte surtout l'événement même (en l'occurrence l'œuvre exposée), que penser de cette même trace là où devrait s'imposer d'abord - et surtout continuer à le faire - la présence d'un lieu, celui de l'exposition, déplacé, concurrencé, bref «ex-situé» par l'ouvrage chargé d'en garder le souvenir?

À cette difficulté, les solutions proposées n'ont certainement pas manqué, la plus rusée en même temps que la plus logique étant sans doute aucun l'inversion des relations de force entre l'événement artistique et sa trace imprimée. C'est ce qui arrive lorsque installations et happenings divers aspirent non plus seulement à se transformer en livre photographique, mais s'élaborent dès le début en fonction de ce destin-là: puisque l'événement a désormais pour vocation première de devenir photographie, la métamorphose se fera avec d'autant moins d'accrocs que l'œuvre se pense radicalement comme une photographie virtuelle et, partant, s'adapte dès sa conception aux caractéristiques du médium qui la divulguera ${ }^{4}$.

S'agissant de l'art in situ, les enjeux de cette transformation sont toutefois plus complexes, puisque l'effort de transposition doit porter également sur ce qui, par définition, résiste plus tenacement à la reprise photographique que l'œuvre même et que, de surcroît, la solution simple mais 
radicale - le remplacement de l'événement par sa version livresque, soit l'annulation de l'exposition au profit du seul catalogue - lui reste en principe interdite. Certes, on peut choisir comme lieu d'accueil-c'est-à-dire de réalisation - de l'œuvre le lieu même de son archivage, qu'il s'agisse d'un livre, d'un cédérom, d'un site Internet ou d'un autre dispositif cyberspatial, qui tous permettent et la circulation de l'œuvre et son intégration concertée à un endroit spécifique. Mais pareille démarche, quand bien même elle a guidé plus d'une pratique passionnante ${ }^{5}$, évite le problème de la transposition "cataloguée» plus qu'elle n'y offre de nouvelles réponses.

Pour sortir de l'impasse, le mieux est peut-être encore d'exploiter la double logique qui traverse inévitablement chaque catalogue. Oscillant entre document et monument, entre transparence et opacité, entre la trace de l'événement et l'événement lui-même, tout catalogue est en mesure de construire, du moins théoriquement, une sorte de diagramme des rapports entre l'œuvre et son lieu. En effet, s'il reste impensable de reproduire tels quels aussi bien l'œuvre que son lieu d'accueil, la relation entre les deux peut être transposée, avec tout ce qu'une telle traduction implique de torsions, de pertes, mais aussi d'excroissances et de trouvailles inédites, au niveau des rapports entre le nouveau site d'accueil qu'est l'objet-livre, d'un côté, et la version photographique de l'œuvre en question, de l'autre. Dit autrement: sans renier sa nécessaire vocation "documentaire», ni rompre avec ses propres ambitions «monumentales», le catalogue d'œuvre, double ex situ d'un travail in situ, a peut-être les moyens, diagrammatiques en l'occurrence, de rendre compte d'une production in situ ailleurs qu'en son propre lieu de fonctionnement, tout en maintenant l'esprit et surtout la forme de l'entreprise à montrer.

\section{«In situ» et "génie du lieu»}

Kounellis au château de Plieux, le catalogue accompagnant le passage du maître de l'arte povera dans le château-tour de Plieux, un des sites historiques de France les plus ouverts à l'art contemporain ${ }^{6}$, témoigne à bien des égards d'un tel programme, qu'il réalise selon des voies qu'il n'est pas inutile de décrire et d'analyser jusqu'en détail. Non pas pour ériger cette publication en volume ${ }^{7}$, mais afin de mettre en exergue certains des mécanismes pouvant accompagner la continuation et la différenciation ultérieure d'une démarche in situ sous forme de livre.

Le château de Plieux (mais gardons-nous de confondre trop vite le nom commun "château» et le nom propre
«Plieux» avec la construction médiévale qu'ils paraissent désigner sans équivoque) diffère des nombreux autres endroits comparables de France non pas du fait qu'il soit habité (ce serait même plutôt la règle), mais du fait que son habitant, l'écrivain Renaud Camus, soit un de ceux qui ont le plus œuvré pour donner à la notion de "génie du lieu» un sens nouveau. Sous peine de caricaturer ici une pensée et une attitude fort complexes, on peut essayer de circonscrire comme suit ce réseau de significations, d'attitudes, de sensations, de rêves et de désirs. Loin de limiter la notion de génie du lieu à la rencontre d'un certain éclat, celui de l'endroit visité, et d'une disponibilité exemplaire, celle du sujet ouvert à l'épiphanie du lieu, les textes de Renaud Camus proposent une singulière radicalisation de pareille fusion entre sujet et objet. D'un côté, en effet, l'auteur pousse l'union à un degré que la modernité, avec sa méfiance de l'illusion référentielle, avait rejeté: chez Renaud Camus non seulement les mots et les choses parfois communiquent, mais dans certains cas ils finissent par se faire indissociables. Comme il est écrit dans Le Lac de Caresse ${ }^{8}$ : « [...] car si désincarnés que soient le livre à faire et ses mots, ils ne sauraient se résoudre à n'avoir pas de répondant sur la terre» (p.60). D’un autre côté, pourtant, presque toutes les lignes de ce même ouvrage et de bien d'autres gardent aussi conscience de la non moins inéluctable séparation des pages et des paysages, pour reprendre une paronomase chère à l'auteur qui ne rompt nullement avec les acquis de la modernité d'inspiration mallarméenne. La scission, toutefois, n'est pas toujours vécue tragiquement, mais au contraire revendiquée comme une des garanties de la distance entre l'homme et les choses, entre l'homme et les autres ou encore entre l'homme et soi-même, sans laquelle nulle vie et nulle respiration ne seraient possibles. De ce point de vue, les plus belles épiphanies sont celles qui débouchent sur le génie d'un non-lieu, d'un lieu vidé, séparé de lui-même par l'action des signes empêchant la redite mortifere, la répétition tautologique, l'étouffante orthodoxie.

S'agissant du château de Plieux, ce désir de créer une distance entre le lieu et son nom surgit dès les premières phrases du catalogue. Par tout un réseau d'associations de formes et de thèmes, le substantif "château» s'allégorise et devient comme un reflet du nom propre qu'est le Château de Kafka (le patronyme de Kounellis est rapproché de celui de K. dès le titre du texte d'escorte de Renaud Camus: «K. dans le château, enfin ", la suite de ces mêmes pages permettant de revenir plusieurs fois sur les échanges entre les œuvres de l'artiste et les lettres de l'alphabet). De son côté, le 
toponyme «Plieux» participe de ces va-et-vient entre l'être et les lettres par l'importance des endogrammes que mobilise ce terme dans un contexte pareil: Plieux rime avec «lieu», ou plutôt avec «lieux» (la nuance est de taille pour peu qu'on se rappelle le besoin de distance et de séparation intérieures), tout en rappelant le nom tout mallarméen de l'association qui gère le site, "Pli selon pli».

C'est donc ce lieu-là qui accueille K. ou plutôt le travail de K., lequel intervient diversement pour remodeler l'espace du château. Dans deux salles du bas, avec leur sol en terre battue, sont introduites des poutres et des solives, qui tantôt paraissent soutenir les murs (dans la première salle) et tantôt semblent y reposer (dans la deuxième salle). Dans la grande salle du château, avec son plancher en bois, flottent de grosses pierres, accrochées au plafond par des cordages marins. Au sommet de la tour, enfin, se hisse un drapeau dessiné par l'artiste ${ }^{9}$. Les matériaux utilisés sont simples, comme est claire l'idée porteuse de l'ensemble de cette grande métaphore filée: une progression s'installe qui mène du très lourd au très léger, de la terre à l'air, en passant par le bois et la pierre et les fonctions couplées de l'étayage et du repos, de l'envol et du flottement, le tout pouvant se déchiffrer comme la mutation du château en navire, avec, de haut en bas, la cale du navire, puis le nef et les voiles et, tout à la fin, le pavillon de l'embarquement.

Toute la question est maintenant de savoir si pareille organisation se retrouve sans altération dans le catalogue ou si, au contraire, la version livresque du travail de K. refaçonne son idée tout en la documentant.

Apparemment, la segmentation des pages du livre épouse sans trop d'écarts la division interne du trajet de l'exposition. Sans être pour autant séparées de manière catégorique, les quatre parties de l'intervention de Kounellis se reconnaissent d'emblée, elles ne se mélangent pas et leur division est rehaussée par la répartition calculée des photos couleur: l'enfilade d'images en noir et blanc est ponctuée par quatre hektachromes, soit exactement une image couleur par section. De la même façon, l'alternance admise de plages de texte en fausse page et d'illustrations en belle page assimile, sans trop de peine, la traversée du livre à une sorte de tour guidée.

Or, en dépit de cette correspondance indéniable, la présentation du catalogue paraît plus complexe que celle offerte par l'exposition. Si on commence par examiner l'ordre d'apparition des fragments retenus, trois caractéristiques sautent tout de suite aux yeux: d'abord la divulgation du clou du spectacle en première de couverture; ensuite l'in- sertion d'un ensemble périgraphique à la fois semblable au reste du livre et fort différent de lui ${ }^{10}$; enfin les variations internes d'une séquence à l'autre. À l'exception du premier trait, qu'il est possible de présenter brièvement, on s'efforcera d'analyser avec toute la minutie requise ces propriétés importantes du catalogue Kounellis au château de Plieux.

\section{La couverture}

L'exhibition des "pierres flottantes» en recto de couverture, le premier trait à commenter, est-elle là pour vendre la mèche avant que l'exploration du château même n'ait commencé pour de bon? Serait-elle dictée par des considérations mercantiles destinées à aguicher le lecteur pressé? L'une comme l'autre de ces explications paraissent bien minces. Plutôt convient-il de supposer que la brusque inversion de l'ordre des choses, le livre commençant in medias res là où le parcours réel de la visite est nécessairement linéaire et sans doute aussi fléché, ouvre le regard à ce qui, dans la visite "sur papier», va échapper à la contrainte de la ligne droite. Cette lecture, plus satisfaisante par rapport au travail de Kounellis, s'avérera en tous points très rentable lors du voyage dans le livre même.

\section{L'intermède périgraphique}

La seconde surprise disposée par le catalogue, l'élaboration d'un intermède péritextuel d'un raffinement extrême ${ }^{11}$ (voir illustrations 1 à 3), fait succéder à la vue panoramique du château de Plieux le gros plan d'un dessin préparatoire de Kounellis, avant de caler le regard au pied d'un édifice occupant presque tout le champ. Le bâtiment paraît s' élever vers le drapeau au sommet où se retrouve, mais "mis en vie» 12 , le croquis antérieur. Cependant, pour étudier correctement les rapports entre ces trois états du péritexte ${ }^{13}$, il ne suffit pas de signaler que tout l'enchaînement se fonde sur le principe de l'avant et de l'après (l'image centrale qui affiche le travail de Kounellis étant l'axe temporel sur lequel pivote la scène), il faut dépasser cette logique très linéaire qui ne distingue en rien l'avancée sur le terrain (où on se rapproche du château) et celle dans le livre (où on tourne les pages les unes après les autres).

En examinant de plus près le rapport entre les deux premières images, on constate tout d'abord la bipartition franche de la feuille, avec il est vrai un renversement tout aussi direct. L'esquisse de Kounellis est cadrée et montrée de manière à correspondre aussi exactement que possible à l'emplacement du ciel noir de la première photographie ${ }^{14}$ 

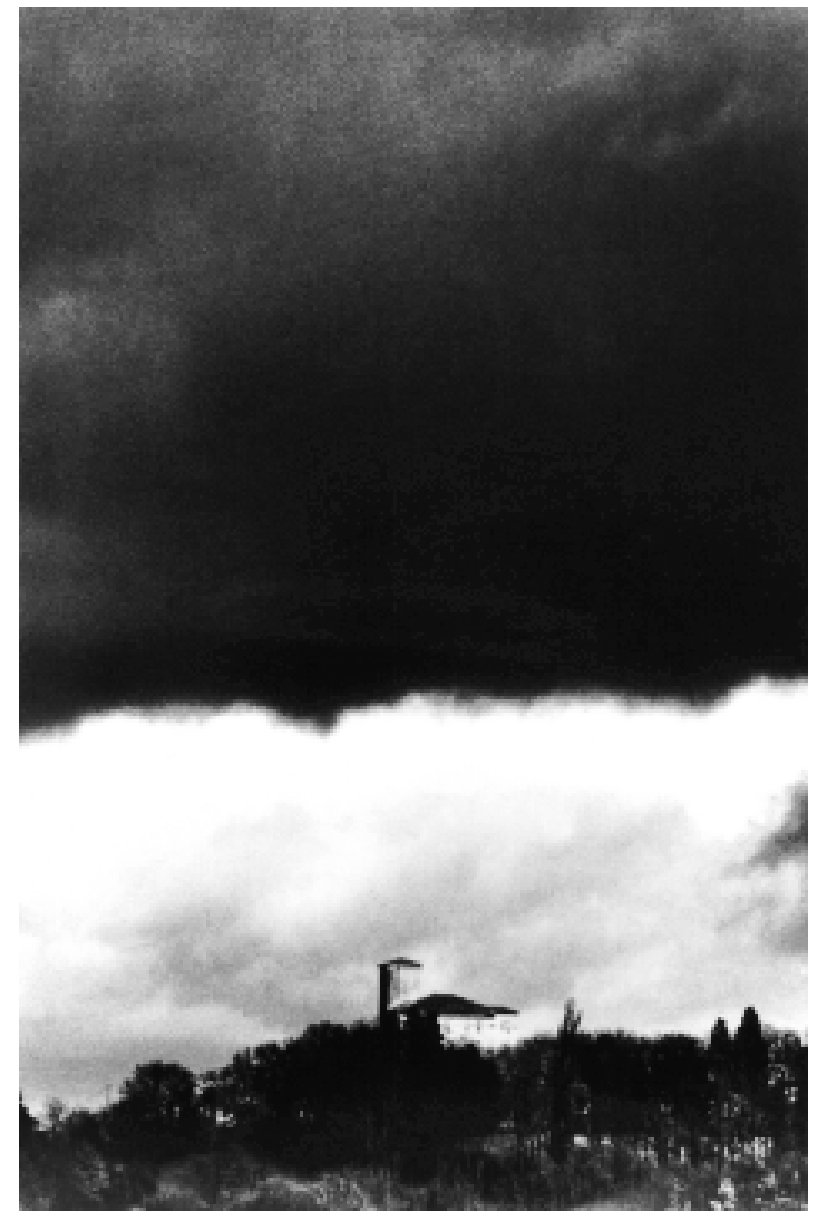

Illustration 1

Nature et culture sont ainsi superposées dans le haut de l'image, cependant que le bas, envahi tantôt par le château et tantôt par le blanc du support, semble associé à un vide, à une manière de non-lieu. Par-dessus le marché, l'intervention d'un creux et le recours à la permutation inscrivent ces pages dans la perspective d'une combinatoire, d'une logique sérielle susceptible de se saisir également des autres paramètres montés en épingle par les ruptures d'une page à l'autre: l'extrême changement d'échelle (après la vue panoramique, on trouve un très gros plan), l'orientation des lignes de force (les horizontales se compliquent d'une verticale) ou encore l'hésitation persistante entre abstrait et concret (la croix dessinée par Kounellis, c'est d'abord deux lignes qui se coupent, mais c'est aussi, par l'effilochement même du trait, un rappel prégnant des lourds nuages ouatés).

Si nets sont les rapports différentiels entre les deux premières images que leur structure en devient dynamique.

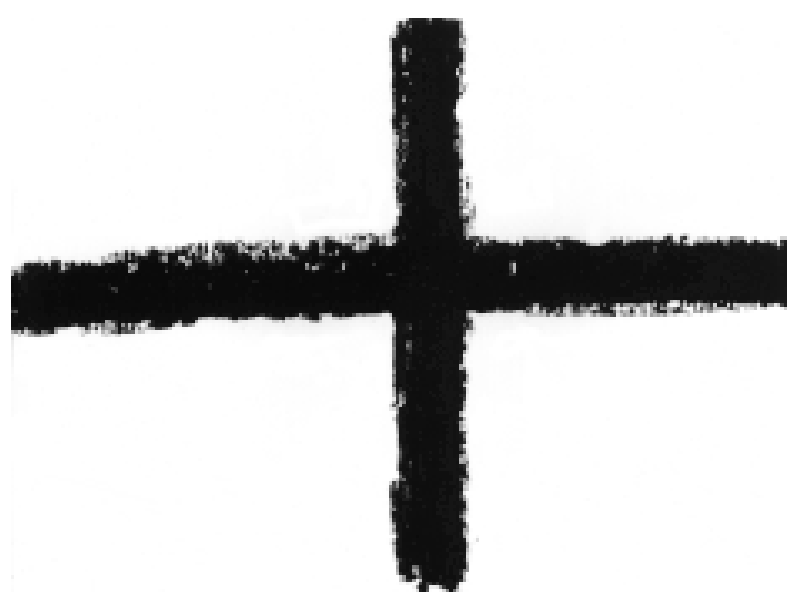

Illustration 2

Non seulement les possibles ne paraissent guère épuisés par les deux premières occurrences de la série, mais ils sont surtout en partie prévisibles, ce qui va installer dans le catalogue une relation nouvelle avec le lecteur. À l'instar du parcours dans les lieux réels, la progression dans le livre prend en effet la forme d'un défi lancé au lecteur, qui peut rivaliser avec l'auteur comme dans un roman policier, en essayant d'anticiper sur les déroulements futurs de l'ouvrage.

Par rapport à cet horizon d'attente, il y a lieu de décrire la troisième image comme le dernier volet d'un triptyque. Ce sont, en effet, les mêmes paramètres que la photographie et sa mise en page mettent à contribution, mais jamais de façon mécanique: l'inversion des valeurs chromatiques fait qu'après le passage du noir au blanc on aboutit à la domination d'une gamme de gris; le découpage horizontal de la feuille en deux moitiés plus ou moins égales se retrouve dans la division comparable de l'édifice en corps central et en tour, les deux agités de lignes obliques jusque-là absentes ${ }^{15}$; le rapport quantitatif entre le léger et l'évanescent, d'une part, et le compact et le solide, d'autre part, se conserve: les nuages ne surplombent plus le château mais le vent reste visible dans les plis de l'oriflamme; le maintien du noir avait rendu attentif aux similitudes matérielles entre la représentation des ciels et les effilochures du dessin de Kounellis, que ne contredisent pas dans l'image finale les camaïeux gris des murs; enfin, la césure entre l'état naturel et l'état culturel des lieux est intériorisée, le drapeau occupant par rapport au château ainsi «naturalisé» la même place que le château par rapport à l'immensité du paysage gersois, il est vrai à un endroit symétriquement inverse de la page, ici tout en bas et là tout en haut. 


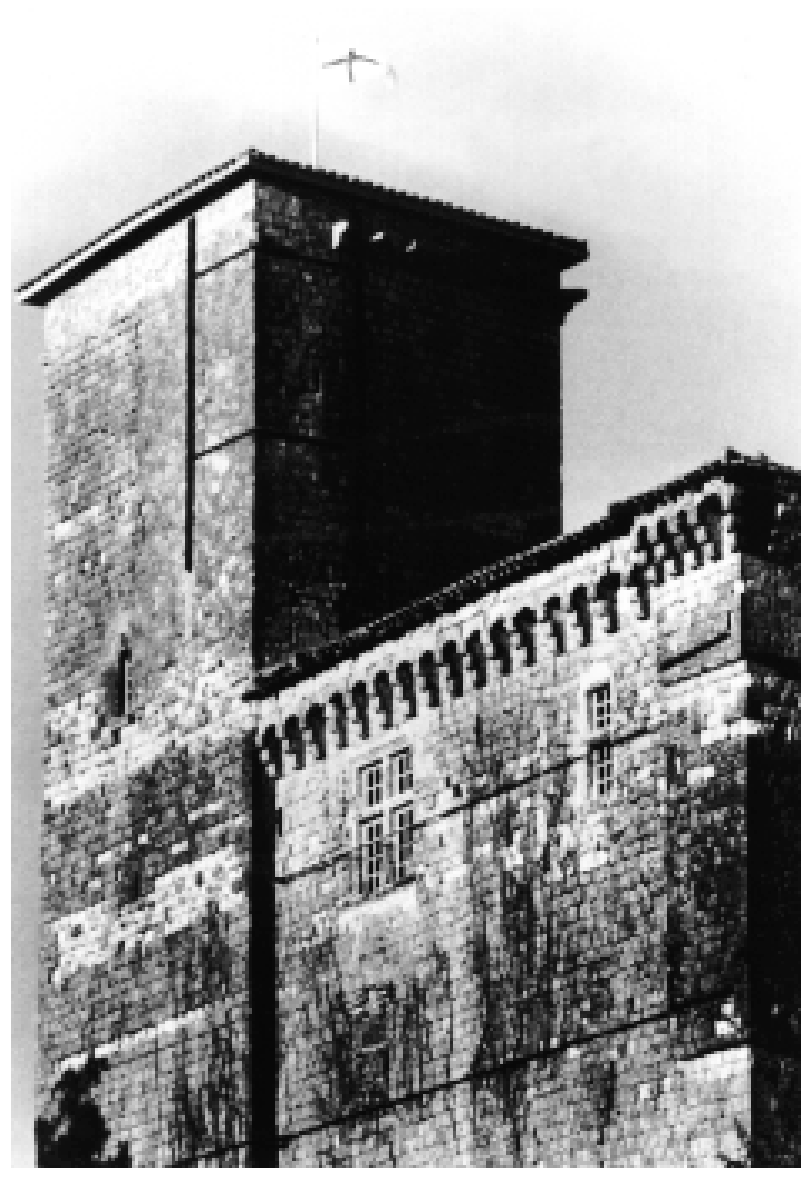

Illustration 3

Dans ce vaste dispositif, l'esquisse de l'oriflamme n'a pas de fonction univoque. À cause de son contenu et de sa forme spécifiques, la croix est, en effet, à lire d'abord comme le symbole de la copule (la réduction du pavé imprimé corroborant le statut à part de l'élément central du triptyque). Ensuite pourtant, lorsqu'apparaît la réalisation de l'esquisse sous forme d'oriflamme, la même croix est aussi, non plus le conjoncteur, mais le terme qui s'ajoute lui-même à l'image inaugurale du château. Ainsi on cesse d'avoir affaire à une structure simple $" A+B=A B »$, pour déboucher sur une structure du type $« \mathrm{~A}+($ symbole de conjonction $)=\mathrm{A}$ trans formé», logique nouvelle qui court-circuite les instances habituelles et assigne la fonction de copule à autre chose que ce qui l'explicite, c'est-à-dire la croix. L'élément conjoncteur devient alors l'organisation interne du catalogue même, avec son enchaînement d'illustrations en belle page qui déteignent l'une sur l'autre par empilement et presque par transparence. Comme ce genre de superpositions ne peuvent exister que dans le livre, elles démontrent à quel point la visite de l'exposition est ici liée à la forme du support-livre, le catalogue ménageant des raccourcis et des alliances autres que ceux agencés par le travail premier in situ.

À côté de ces nombreuses correspondances d'une image périgraphique à l'autre, frappent pourtant aussi certaines différences. Leur intérêt est d'autant plus grand qu'il s'agit de glissements concentrés sur les parties les plus stratégiques ou surdéterminées de l'image: l'oriflamme et le château. L'une et l'autre se dérobent, en effet, à la réticulation par lignes horizontales et verticales, si massivement présentes dans les images d'ouverture. La forme triangulaire de l'oriflamme est soulignée par l'action du vent. Et ce point de vue, de biais et en contre-plongée, introduit dans la représentation du château une série d'obliques et un angle plutôt aigu, qui tous atténuent la verticalité de la tour et la masse horizontale du corps central de l'édifice.

La géométrisation assez poussée des lieux et des objets photographiés, ainsi que le commentaire de Renaud Camus en regard des images, quand bien même cette voix d'escorte traite surtout des salles montrées seulement plus loin, autorisent en tous cas une lecture "grammatisante» de la photographie. Dans les volumes que reproduit la caméra, on a tout loisir de reconnaitre déjà les lettres grecques qui inspireront si visiblement les formes et emplacements des solives placées dans les salles du bas:

Le tau, dit K, Le pi. Le kappa... Non, il n'a pas dit le kappa; mais la première pièce en entrant, à droite, dans la première salle du bas, à Plieux, a bel et bien la forme d'un K. - retourné ou non selon les points de vue. Quant à la croix centrale, dans la même salle, le maître trouverait ridicule, "redondant", qu'elle fùt sommée. C'est bien un T. De même que la pièce du fond, à gauche, est incontestablement un $\pi$. À quels dieux farouches ces figures majestueuses et si simples, pour le coup, sont-elles des autels? Aux dieux de la lettre, probablement. (p. 14)

Toutefois, plus important encore que cette reconnaissance d'un possible référent scriptural des données plastiques, est le fait que l'association du texte et de la vue amène le regard -invité à balayer la page de gauche à droite par l'insistance sur les couches horizontales - à rapprocher les plages photographiques du catalogue des pavés imprimés. L'opération ne va pas sans surprise et confirme largement le travail général effectué sur les termes-forme et signification confondues que sont «K.», «château» ou «Plieux». Prenons, par ex- 
emple, la légende identifiant la première image de la série: "Château de Plieux (F.S.P.)", où il va aussi sans dire que l'abréviation du nom du photographe contribue à aiguiser notre sensibilité au poids de la lettre. Pour transparent que soit cet énoncé, la continuation horizontale du regard vers la page de gauche prolonge ce bout de phrase d'une remarque sur le nom de Kounellis, dont l'incidence sur la lecture de celui de Plieux n'est pas négligeable. Après avoir souligné la renommée universelle, dans certains milieux, de l'artiste in residence, Renaud Camus poursuit ironiquement:

$O r$, dans le même temps, mais dans d'autres sociétés, plutôt plus nombreuses, à peine fait-on la même annonce on s'aperçoit qu'on suscite, en réponse, à peu près autant d'émotion que si l'on avait dit Ellis Koun, Sillenouk ou Kunö Seïs. (p. 8)

Sous peine de mal lire l'enseignement des anagrammes de «Kounellis», force est, en effet, d'appliquer le même procédé au toponyme "Plieux» (après tout bien moins connu et pour cela même autrement plus fragile que le nom propre de l'artiste aux vertiges des signifiants). Les associations avec «lieu» ou «pli selon pli» se voient ainsi activées une seconde fois, avec du reste une bonne dose d'humour qui enlève à l'exercice tout soupçon de pédanterie. En l'occurrence, et par extension, il n'est pas interdit de remarquer dans les manipulations audacieuses de la distance à l'intérieur de la série d'images du château de Plieux, les vues de loin et de très près s'écrasant presque l'une l'autre, un assez exact équivalent visuel du brassage des lettres du nom de Kounellis/ Plieux.

Plus fondamentalement, ce qui transparaît dans la lecture conjointe d'une séquence photographique d'un château et d'un bloc d'anagrammes d'un nom d'artiste, c'est bien sûr l'union du sujet et du lieu, de l'homme et de la géographie, bref de la personne et d'une certaine idée de l'espace et du monde. Réalisée une première fois in situ par le travail de Kounellis, la même structure va se répéter, d'une manière à peine différente, par l'agencement du catalogue avec des moyens qui lui sont tout à fait spécifiques.

\section{Le déroulement interne}

L'analyse de la troisième grande particularité à l'intérieur du catalogue, le traitement dissemblable réservé aux trois salles de l'exposition, permettra de mieux étayer ce type d'interprétation.

S'il a été crucial d'accorder tant de place aux premières feuilles de ce volume, c'est surtout parce qu'en cette zone se négocie le régime de lecture du livre entier. La primauté, que le catalogue donne aux problèmes de prise de vue et de mise en page, avère le mieux sa véritable portée au moment où s'entame la présentation de l'exposition proprement dite, soit le travail sur les poutres, les pierres et les cordages. $\mathrm{Vu}$ de loin, par exemple après une lecture hâtive des pages d'ouverture, on pourrait avoir l'impression d'un échelonnement très classique, voire trop sage du matériau iconographique, distillé feuille après feuille selon les règles les plus académiques qui soient: une page couleur par salle, assortie d'un nombre variable de photographies en noir et blanc; différences marquées entre les séquences; soulignement des transitions par le recours à des intermèdes dessinés. Pourtant, aguerri par l'activité formelle des premiers segments du livre, le regard du lecteur se fait vite attentif à toute une série de menues interventions visuelles du photographe et du metteur en page qui démontrent amplement le caractère indépendant de la «forme-catalogue» de l'exposition.

Essentiel à cet égard est, bien sûr, le jeu avec la quantité d'information livrée au spectateur, toute différente d'une séquence à l'autre. Non pas au niveau du nombre de photographies par section (il y a respectivement cinq, trois et quatre images pour l'évocation de chacune des salles), mais d'autant plus à hauteur de la répartition entre vues d'ensemble et vues de détail. Progressivement on passe d'une série entièrement constituée d'images très fragmentaires (en la salle $\mathrm{n}^{\circ} 1$ seules se montrent, l'une après l'autre mais aussi un peu mélangées, les pièces introduites par Kounellis) à une série d'images évoluant elles-mêmes (dans leur présentation de la salle $\mathrm{n}^{\circ}$ 2) de l'aperçu global au fragment isolé, puis à un dernier ensemble de vues sans exception générales. Qu'aucune de ces images ne présente le même point de vue ne doit pas être lu comme la transposition immédiate du trajet du visiteur. Ce qui change, en effet, n'est pas d'abord le point de vue, mais surtout la hauteur, la distance, l'éclairage ou d'autres paramètres encore, que ne motive guère la position changée du visiteur. Le parcours est photographique et livresque. Il n'épouse pas le trajet d'une vraie visite. Par moments, il devient même acte d'écriture, car pour saisir correctement la place respective des pièces de la salle $n^{0} 1$ les unes par rapport aux autres, le lecteur est obligé de faire un petit croquis pour y faire figurer l'emplacement des œuvres qu'il ne découvre jamais que par bribes. Dans cette perspective, faut-il y insister, l'allure alphabétique ou scripturaire des pièces exposées accentue encore la transformation d'un cheminement en écriture. 
Le catalogue comme lieu d'écriture

Inséparable de la lecture, le mouvement de l'écriture fait quelque chose que l'image seule ne fait pas (ou ne saurait même pas faire). C'est aussi en ce sens qu'il convient d'entendre l'anecdote suivante, voluptueusement consignée par Renaud Camus dans son commentaire du séjour de Kounellis:

(K. m’offre un dessin, qui représente un Christ, très expressionniste de facture, tordu sur le grand $T$ de la première salle du bas, à Plieux. "On pourrait peut-être le mettre dans le catalogue, dis-je naïvement. - Non, dit K., il faut que ce reste un secret»). [...] «Qu'y a-t-il de plus simple qu'une croix, interroge K., pouvez-vous me le dire? Une chose linéaire, mystique: parfait symbole d'humanité." Puis il demande que les derniers mots soient retirés à l'impression, comme étant «trop explicites». (L'écrivain, Dieu merci, est celui qui trahit les secrets - au bénéfice du Secret, il faut l'espérer [...].

(p. 14, souligné par l'auteur)

En révélant dans son commentaire l'existence d'un dessin interdit de catalogue comme en divulguant des propos voués au silence, l'écrivain resignale de son côté les privilèges du texte et surtout du catalogue, lequel excède aussi l'œuvre dont il n'est censé renfermer, dans la version classique des choses, qu'une pâle copie.

Comme on l'a déjà fait remarquer, le nombre d'images par séquence obéit largement à des soucis d'individuation

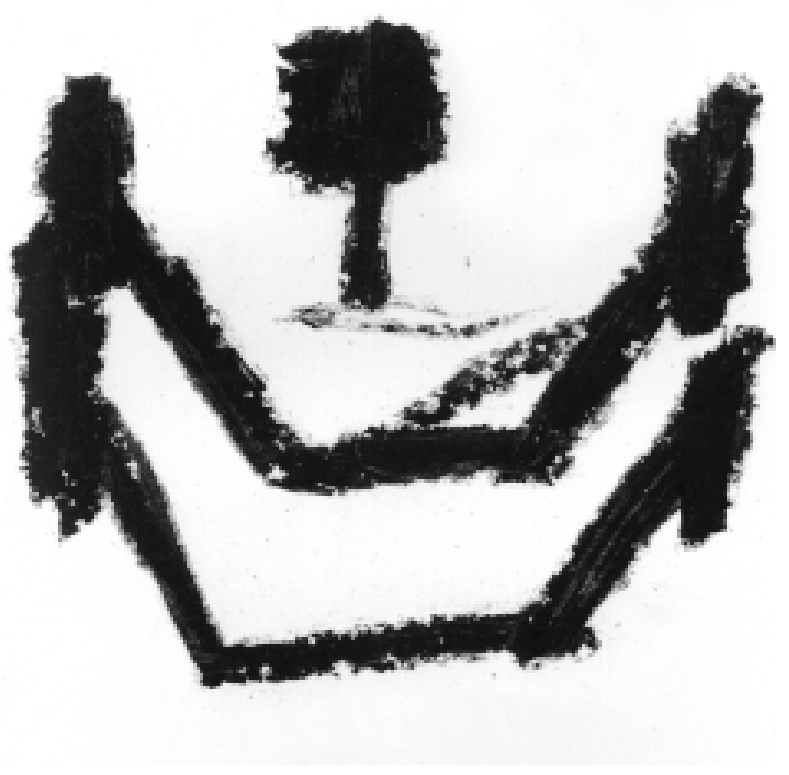

Illustration 4

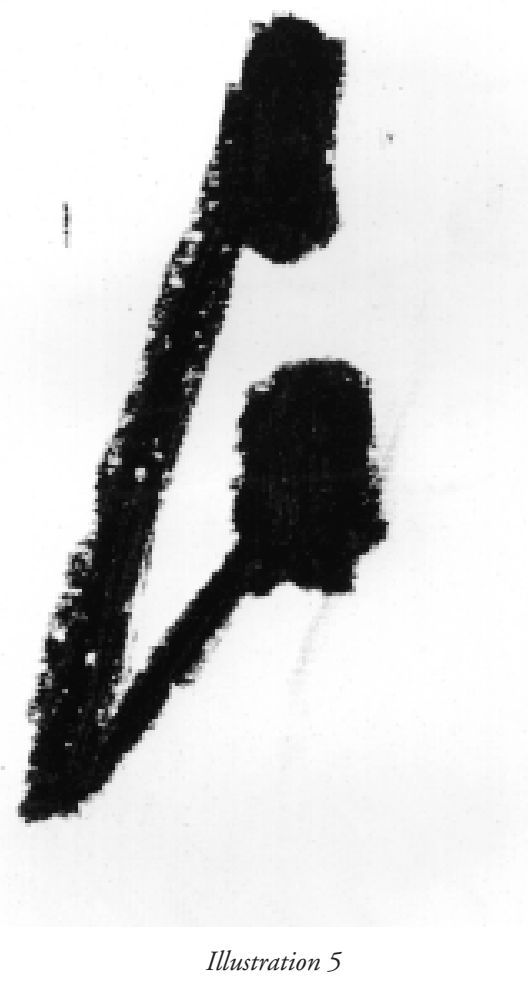

rhétorique. Si on trouve d'abord cinq, puis trois, enfin quatre photos par salle, c'est avant tout pour rendre compte avec précision de la quantité de pièces par salle. En tous cas, c'est la conclusion banale qu'on pourrait tirer de telle variation élémentaire, si le photographe s'était borné à chaque fois à détacher une pièce de son contexte. Or, c'est le contraire qui se produit: souvent, l'image combine des portions dépareillées de plusieurs pièces, à tel point que dans la salle $\mathrm{n}^{\circ} 1$, par exemple, dénombrer les parties constitutives de l'exposition n'est guère chose aisée. Dans la salle du haut, c'est en revanche la nécessité d'une quadruple image, presque identique d'une occurrence à l'autre qui pose problème. Ainsi est mise en cause la représentation transparente qui unit généralement la photographie d'exposition à l'objet reproduit. Ici, chaque image se donne pour un état métonymique du travail à illustrer: une interprétation partielle, provisoire, d'un point de vue lui aussi toujours changeant.

Ce qui encourage également à mettre en avant le catalogue comme principe de transformation et comme producteur de trajectoires inédites, ex situ, dans l'œuvre in situ, est une dernière des grandes propriétés spécifiques de Kounellis au château de Plieux: le statut non identique des esquisses 
insérées entre les trois séquences kounelliennes (voir illustrations 2, 4 et 5). Une fois encore, il pourrait s'agir d'un détail infime; mais, de nouveau, ses conséquences sont tout sauf négligeables. De la première esquisse, on avait déjà fait ressortir le caractère ambivalent. Les deux croquis suivants, qui montrent dans le même style "pauvre» certains détails du projet réalisé ultérieurement, sont placés à la lisière des grandes articulations thématiques du catalogue: l'esquisse $\mathrm{n}^{\circ} 2$ apparaît entre la série consacrée à la salle $n^{0} 1$ et celle consacrée à la salle $n^{\circ} 2$, et l'esquisse $n^{\circ} 3$ sépare ce dernier ensemble des images de la salle du haut. Dans l'un et l'autre cas, leur statut temporel est complexe: le croquis $\mathrm{n}^{\circ} 2$ anticipe sur une série à venir, mais clôt aussi, par sa proximité matérielle, la déclinaison des fragments de la salle $n^{\circ} 1$ (une suite continue de belles pages les réunit, alors que la nouvelle série de la salle $\mathrm{n}^{\circ} 2$ ne débute qu'après une interruption); quant au croquis $n^{\circ} 3$, il suit et couronne l'ensemble de la salle $n^{\circ} 2$, sans rien annoncer de ce qui va suivre. Pareille volatilité temporelle pousse bien sûr à délaisser la seule progression linéaire et fait relire dans l'image de nouvelles exploitations du «sous-texte» (si l'on peut dire) grammatique ou scripturaire du visuel. Hésitant entre relance et clôture, les traits élémentaires des esquisses cessent de représenter uniquement des poutres, afin de se muer en signes de ponctuation - parenthèses tournées de 90 degrés? accolades un rien écrasées? -, symbolisant sans exception une idée d'ouverture fidèle à l'esprit de Kounellis travaillant au château de Plieux.

Le catalogue réitère ainsi à son niveau, dans son espace, dans sa chronologie, le geste créateur de K. L'ex situ, de cette façon, n'est pas moins radical ou novateur que l'in situ - ce qui reste le plus bel hommage que l'on puisse rendre à ce type de manifestation.

\section{NOTES}

1. Voir par exemple T. de Duve, Nominalisme pictural. Marcel Duchamp, la peinture et la modernité, Paris, Minuit, 1984.

2. Pour une introduction fondée théoriquement, voir M. Gauthier, Les Contraintes de l'endroit, Paris, Les Impressions nouvelles, 1987, et G. Lelong, Des Relations édifiantes, Paris, Les Impressions nouvelles, 1991 (le premier volume est axé surtout sur les arts plastiques, l'objet du second est plutôt l'architecture).

3. On peut nommer, en vrac et sans nulle volonté d'être exhaustif, les facteurs suivants: l'internationalisation du marché, qui oblige à se faire connaître ailleurs que là où l'on expose; la pression institutionnelle, qui amène à penser chaque exposition comme une opération de marketing; la domination persistante du discours d'escorte sur l'œuvre elle-même, qui renforce de toute évidence le devenir photographique des produits artistiques.

4. Voir la contribution de P. Dubois, "Pratiques contemporaines de la photographie", à l'ouvrage collectif Histoire de la photographie, dirigé par J.-C. Lemagny et A. Rouillé (Paris, Bordas, 1986).

5. Pour quelques exemples, voir L'Art conceptuel, une perspective, Paris, Musée d'Art Moderne de la Ville de Paris, 1989 (catalogue de l'exposition dirigée par S. Pagé).

6. L'exposition a duré du 15 juillet au 18 septembre 1995. Sur le "fonctionnement» de Plieux, voir R. Camus, P.A. (petite annonce), Paris, P.O.L., 1997.

7. Pour cela, le présent catalogue reste encore trop proche des modèles convenus, qui risquent peut-être d'en faire méconnaître certains aspects des plus originaux.

8. Paris, P.O.L., 1991. Pour un premier commentaire de ce volume, voir notre article "Renaud Camus et le texte sur rien", Texte, no 11, 1994.

9. Non sans clin d'œil, peut-être, au pavillon «bleu blanc jaune» de V. Larbaud, un des auteurs favoris de R. Camus, qui est un écrivain sachant célébrer ses collègues. La suite de l'analyse indiquera pourtant que cette anecdote, assez probable pour qui connaît un peu l'œuvre de Camus comme celle de Larbaud, n'épuise en rien le sens de ce drapeau.

10. Rappelons que le concept de périgraphie, introduit par A. Compagnon dans La Seconde Main (Paris, Seuil, 1979), a servi de base à la notion genettienne de péritexte ( $c f$. G. Genette, Seuils, Paris, Seuil, 1987). On considérera ici les deux termes comme synonymes, étant entendu que les éléments qui, dans l'espace du livre, entourent l'œuvre proprement dite peuvent être de nature aussi bien iconique que scripturale.

11. Dans son $A B C$ du métier, Paris, Imprimerie nationale, 1988, Massin nomme ce genre d'introductions visuelles au texte des "déroulements » et les compare très justement à l'ouverture d'une symphonie.

12. Empruntés à J. Ricardou, Le Nouveau Roman, Paris, Seuil, coll. "Points", 1990, les concepts de "mise en vie" et de "mise à mort" désignent respectivement le passage d'une représentation fictive à un référent réputé réel et le passage d'un référent réputé réel à une représentation fictive.

13. Pour autant bien sûr que les plages périgraphiques en question soient séparables de l'œuvre même. Le critère de séparation utilisé n'a pas trait à l'opposition intérieur/extérieur du château (le pavillon dessiné par Kounellis ne fait pas moins partie de l'exposition que ce qui se montre dans les trois salles), mais concerne le fait autrement plus matériel de l'unicité des images: là où chacune des salles bénéficie de plusieurs prises de vues, les images de l'ouverture péritextuelle montrent toutes des objets fort différents. 14. Une comparaison avec les deux autres croquis de Kounellis montrés ultérieurement laisse supposer nettement que la page de dessin a été recadrée en vue de l'obtention de l'effet mentionné ( $c f$. infra pour une reproduction de ces esquisses).

15. Le déplacement latéral du champ de fuite accentue évidemment l'impact des lignes obliques présentes dans le champ. 